\title{
Estrategias prácticas de gestión de contenidos
}

\section{Por Andrew Cox y Jane Yeadon}

Resumen: Descripción de métodos para la gestión de contenidos de servicios de información con un enfoque práctico y de «bajo nivel tecnológico», como la formación de usuarios en html, o haciendo que éstos usen plantillas, así como analizando estudios de caso de bdds vía web y sistemas gestores de contenidos.

Palabras clave: Gestión de contenidos, Gestión de la información, Servicios de información, Sistemas gestores de contenidos, Estudios de caso.

\section{Title: Content management: practical strategies}

Abstract: A range of content management strategies for information services is described, stressing practical and «low tech» approaches such as training users in html or providing them with templates to work with, as well as looking at case studies of databases available over the web and true content management systems.

Keywords: Content management, Information management, Information services, Content management systems, Case studies.

Cox, Andrew; Yeadon, Jane. "Estrategias prácticas en gestión de contenidos". En: El profesional de la información, 2002, nov.-dic., v. 11, n. 6, pp. 466-474. 


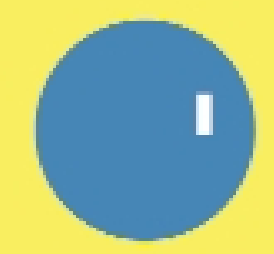

$\circ \quad V \quad I \quad D$

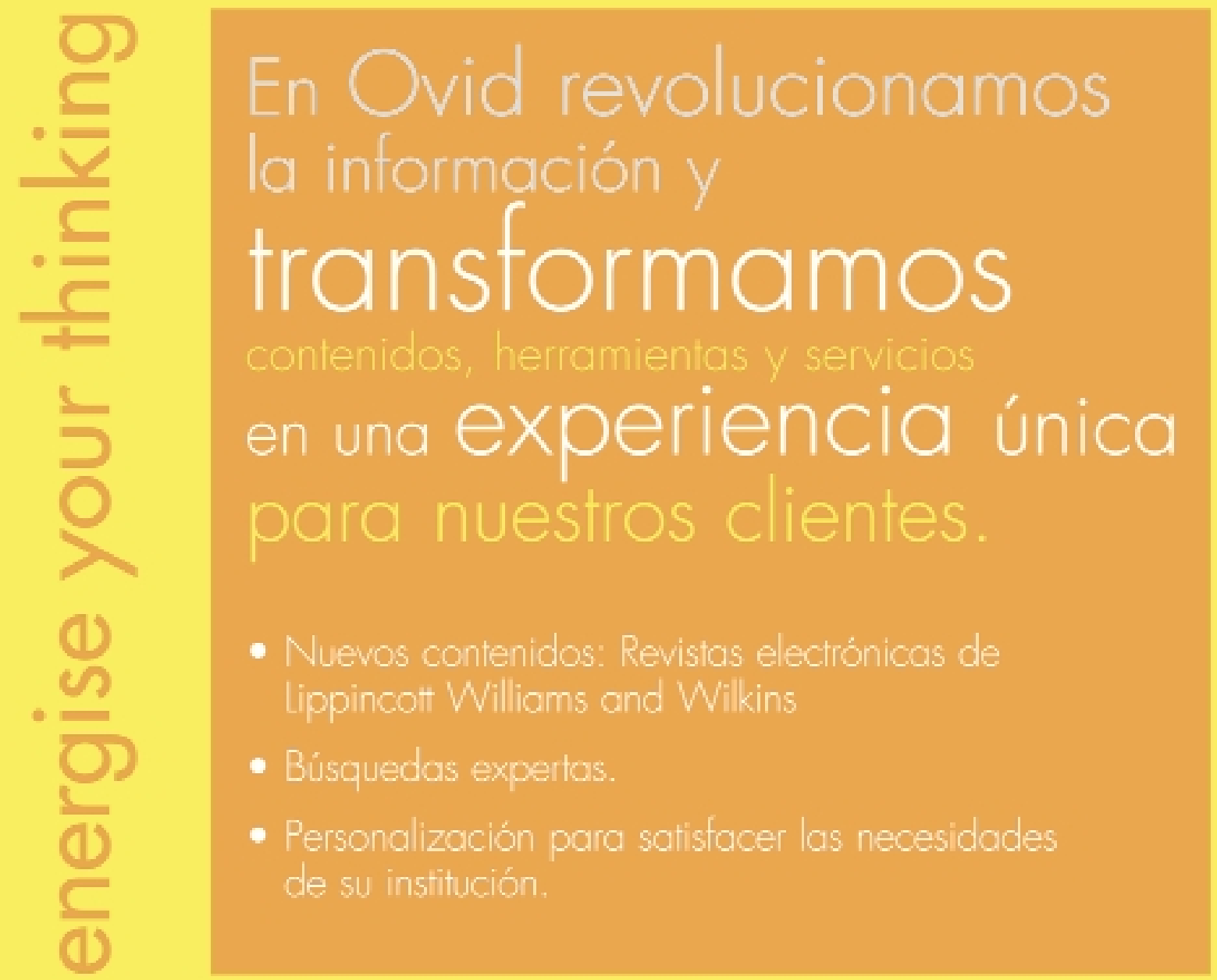

Madrid tél 914186275 Barcelona tél 938106563 spain@ovid.com 


\section{Introducción}

Hace tiempo que se habla de gestión de contenidos... Quizá alguien todavía se pregunte jes lo mismo que gestión del conocimiento? ¿qué relación existe entre ambos conceptos? La diferencia clave, a nuestro parecer y en pocas palabras, es que la gestión del conocimiento se basa en el contenido y el significado de la información, y la gestión de contenidos en el modo de difundirla y presentarla. En este artículo vamos a centrarnos en el segundo de los conceptos.

Todos los sitios web de las organizaciones requieren algún tipo de gestión de contenidos (gc) en mayor o menor proporción dependiendo de su complejidad. A menudo existe una correlación entre la estructura del organismo y la de su web, y esto es cada vez más así puesto que el web se va convirtiendo en «el» escaparate usual del organismo.

Además de las razones obvias de ordenar, clasificar, coordinar, etc., seguramente el mayor beneficio que indirectamente ofrece la gc es estimular la participación y comprometer al personal en la cre- ación y mantenimiento del web, y también la mejora de la precisión y calidad de los contenidos.

La gc es un proceso que se puede implementar a través de políticas de gestión y/o con el soporte de software especializado: los sistemas gestores de contenidos (sgc).

\section{Una buena política es me- jor que un software potente}

Para la mayoría de bibliotecas no es necesario disponer de un software muy elaborado para gestionar los contenidos de su web. Una forma frecuente y suficientemente válida es desarrollar una buena normativa que contemple aspectos tales como:

—quién es el responsable del mantenimiento de las páginas;

-frecuencia con que éstas tendrían que revisarse;

—cómo se tienen que notificar los cambios al webmaster;

- tiempo de que dispone el webmaster para llevar a cabo los cambios;

—requerimientos mínimos de diseño: logos, tipografía, colores

\section{Litc y la South Bank University}

La SBU es uno de los 40 politécnicos - en concreto, el Polytechnics of North London- que con la UK Further \& Higher Education Act de 1992 se convirtieron en universidades.

El Library Information and Technology Centre se originó como proyecto de The British Library adjudicado al PNL, y fue una visita obligada para todos los que por los 80 s empezaban a automatizar sus bibliotecas y deseaban recibir asesoramiento.

Más tarde, y ya en la SBU, Litc se convirtió en una unidad de investigación algo más general desarrollando numerosos proyectos europeos y británicos. Está encuadrado dentro de los Learning \& Information Services, cuyo director es John Akeroyd. Otra conocida personalidad relacionada con Litc es su director adjunto Robin Yeates.

Lisa es el servicio de información de tipo interno (aunque abierto al exterior) de LIS, y no debe ser confundido con la base de datos $\mathrm{Li}$ brary and information science abstracts, con la que no existe ninguna relación.

Desgraciadamente, en el momento de escribir esta nota el futuro del Litc es incierto.

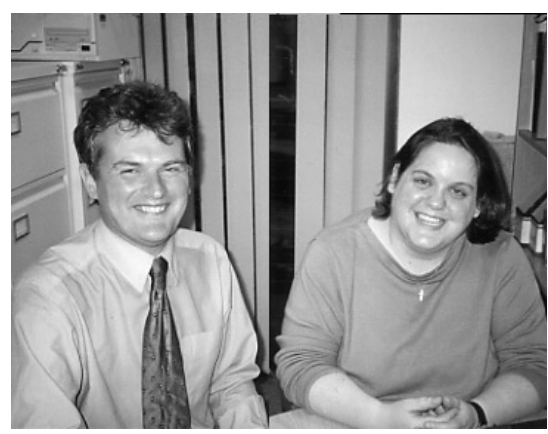

Andrew Cox y Jane Yeadon, Litc, SBU

estándar; poner o no la fecha de la última vez que se modificó la página; cómo y dónde poner el nombre de la persona responsable de la actualización de la página...;

- cómo poner nombre a las páginas y asignar metadatos;

-etc.

La normativa tiene que publicarse internamente para que los usuarios puedan comprobar en todo momento que se ajustan a ella; en caso de que haya más de un editor de páginas (cosa frecuente cuando cada departamento se encarga de la información contenida en su respectivo directorio del web) una política y un protocolo de procedimiento escritos son doblemente esenciales.

\section{Herramientas simples}

Existe una serie de herramientas simples que facilitan mucho la gestión de contenidos en el web. Una aplicación clave es un sistema que permita buscar y reemplazar en múltiples archivos a la vez, de manera que el administrador pueda cambiar las direcciones url (o determinados trozos de texto) en toda el web sin tener que predefinir qué parte tendrá que ser modificada. Normalmente los sgc permiten hacer cambios en los enlaces con sólo un click de ratón, pero esto a menudo presupone que el enlace tiene que haber sido definido como un objeto.

También son útiles herramientas para la comprobación automática de links, corrector lingüístico, sistemas para validar la correcta 
programación (p. ej., que se cumplan las normas de accesibilidad). Un truco ingenioso es poner un código en cada página que indique cuándo se tiene que revisar, lo cual permite localizar las páginas correspondientes mediante una búsqueda. Por ejemplo, buscando xjun2003 se detectarían las que tienen que revisarse en junio de 2003.

\section{Formación, plantillas y ho- jas de estilo}

Como se ha comentado al principio, uno de los objetivos principales de la gc es implicar al mayor número de personas posible en la creación y mantenimiento de contenidos, sin tener que sacrificar calidad, homogeneidad ni seguridad.

Lo primero que una organización debería plantearse es si quiere que sus miembros tengan control directo en la creación y actualización de las páginas web. Esto implica un riesgo, ya que reduce grandemente el control del webmaster sobre el diseño y la estructura del web. Una política clara podría ayudar a regular esta opción, aunque luego realmente se demuestra en la práctica que el control es difícil.

Otra posibilidad más segura es que los usuarios envíen las páginas modificadas al webmaster, el cual se hace responsable de aprobarlas y publicarlas, pero esto tiene el inconveniente de que a veces corregir páginas exige más trabajo al webmaster que crearlas él mismo. Por otra parte siempre están las personas que se resisten a participar y dejan partes del web olvidadas y sin mantenimiento.

Independientemente de la opción escogida por parte de la organización, es necesaria la formación del personal en editores web y/o clientes ftp. Existen editores wysiwyg (what you see is what you get, lo que ve ahora es lo que obtendrá luego) muy fáciles de manejar, ya que requieren poco conoci-

\section{¿Qué contenidos tienen que gestionar las bibliote- cas?}

Textos, documentos (tratados como objetos), urls, imágenes..., y cada vez más ficheros de sonido y vídeo. Quizá tengan que tratar también otros contenidos más especializados: datos sin elaborar (en servicios de información financiera) o material de enseñanza (en organizaciones educativas). En los servicios de información clásicos el conteniinformación bibliográfica, pero de ella se encarga un sistema de gestión bibliotecaria estándar.

miento de html — por ejemplo Netscape y Explorer los ofrecen gratuitamente-

Algunos programas como DreamWeaver o FrontPage permiten utilizar plantillas, es decir, páginas con características comunes prediseñadas y controladas por el webmaster, de forma que los usuarios sólo tienen que añadir los contenidos. Esto no garantiza que los usuarios no puedan cambiar cosas de la plantilla, pero es un sistema viable en la práctica si el envío por ftp al web sólo lo puede hacer el webmaster. do principal está constituido por

Un método ligeramente distinto consiste en seguir una norma de diseño común e introducir el contenido utilizando una hoja de estilo (sistema también conocido como CSS, Cascading StyleSheets).

Otra forma de definir características principales de una página es a través de los comandos SSI, Sever-Side Includes, es decir, secciones adicionales de html agregadas dinámicamente a las páginas a medida que éstas llegan al usuario del sitio. Un ejemplo son las barras de navegación, las cuales aparecen homogéneamente en todas las páginas, pero de un modo que no se pueden borrar ni modificar.

Es fácil ver por qué los $S S I$ no han tenido demasiado éxito: añaden una capa de complejidad para el diseñador, el cual tiene que pensar en el aspecto que tendrá la pagina final con ellos. Probablemente los SSI tengan que ser sólo una parte muy simple del código, sólo para conferir un aspecto distintivo al texto, o sean una ayuda para la navegación.

Una forma atractiva que parece usarse poco es el formulario web, que permite a las personas autorizadas añadir contenidos. Lo más simple es instalarlo en un boletín

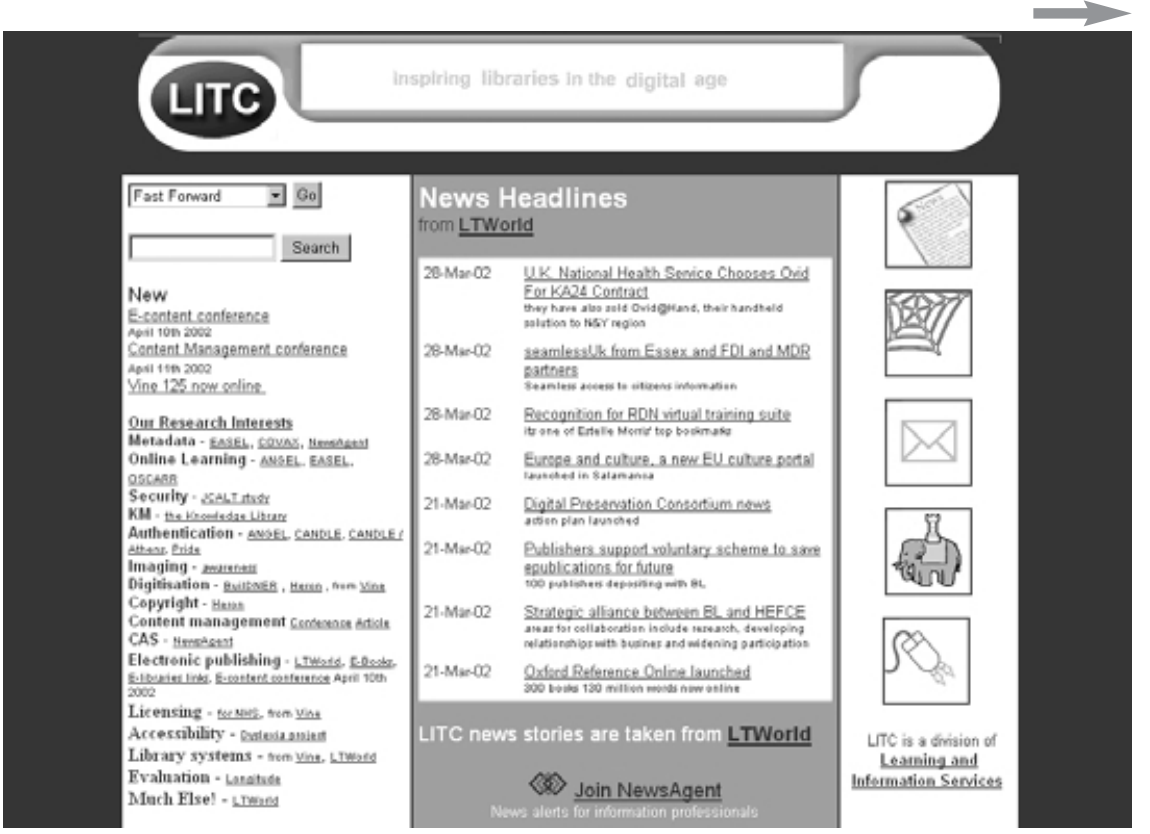

Fig. 1. Portada del Litc 


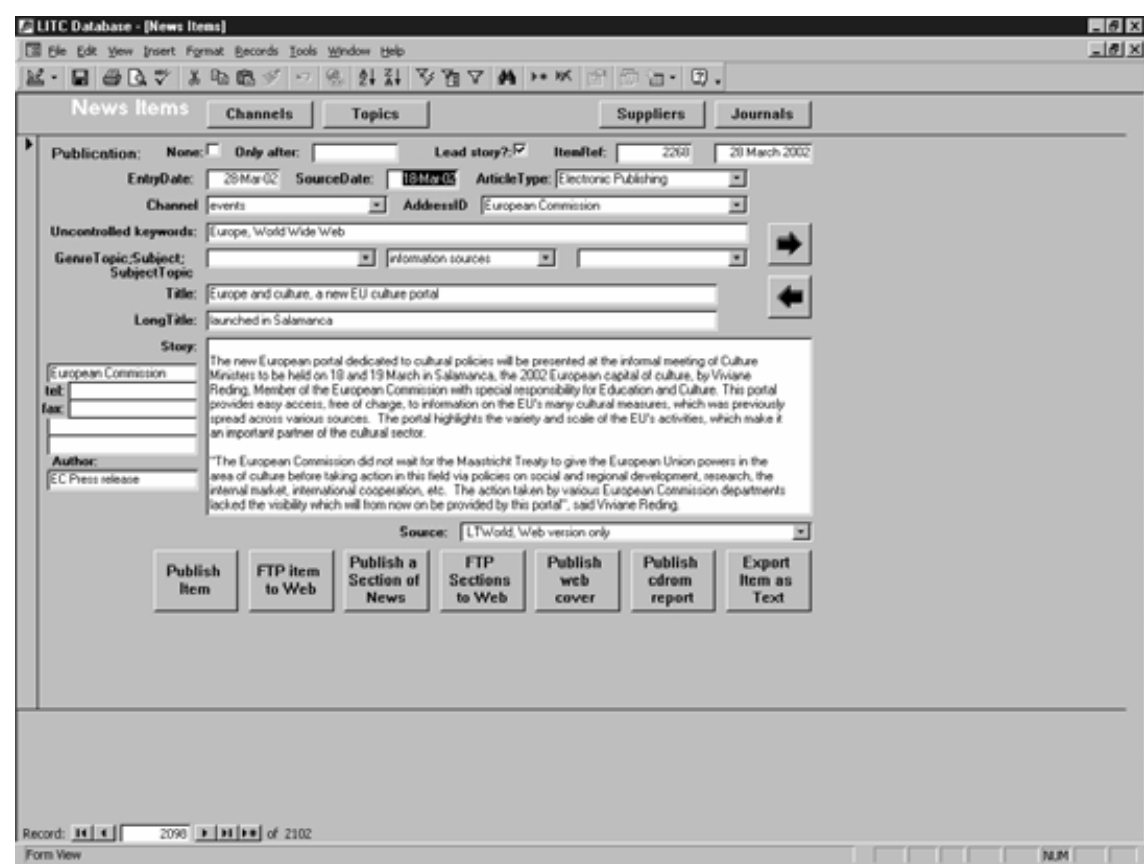

Fig. 2. Formulario en Access para introducir noticias en LTWorld

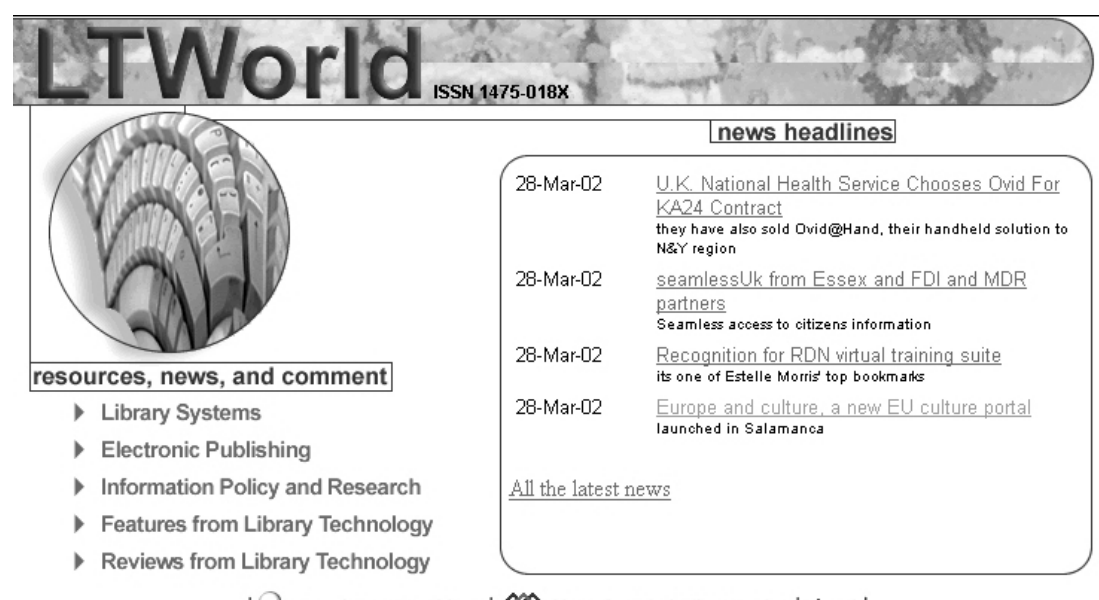

IQ search news archives | NewsAgent alerting service | about |

Fig 3. Portada del boletín-e LTWorld

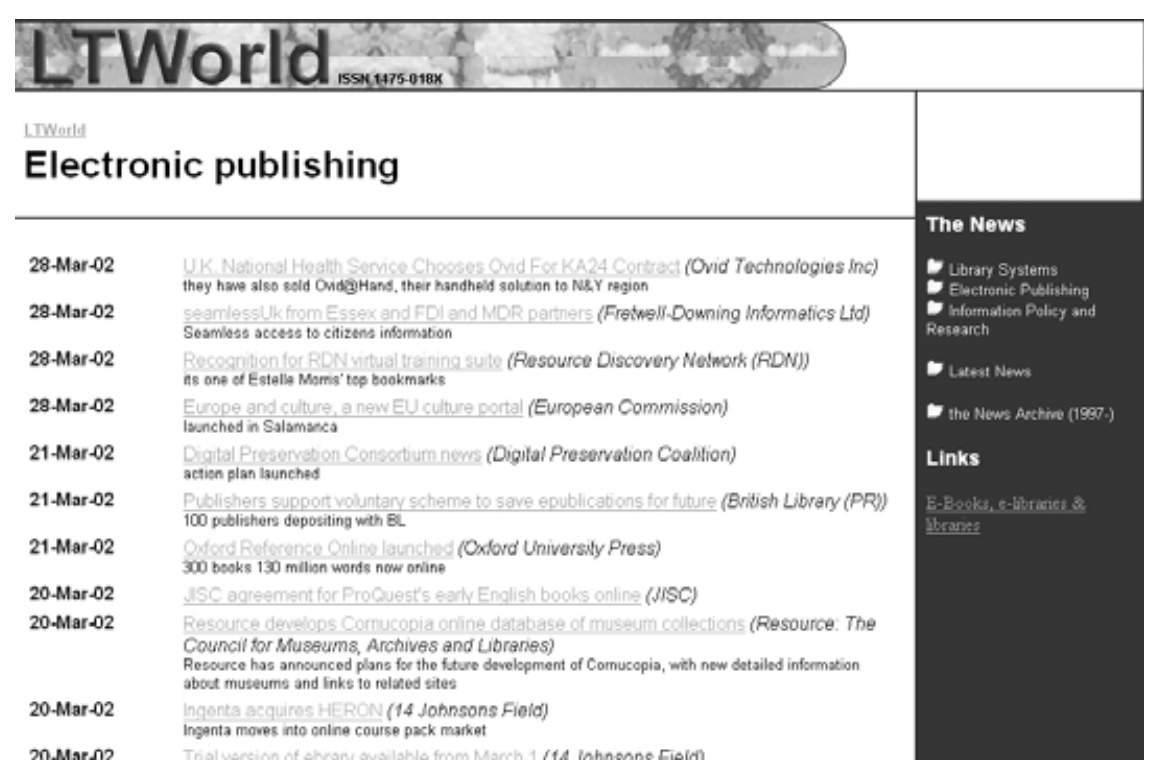

Fig. 4. Página de una sección de noticias -éstas se agrupan por secciones- electrónico, en el cual el usuario sólo tiene que teclear la información y clicar el botón "enviar". Evidentemente no se puede cambiar la estructura predeterminada, y a su recepción el contenido suele quedar ordenado cronológicamente. La única dificultad puede estar en la gestión de las autorizaciones, pero resulta un sistema práctico para añadir noticias o nuevos recursos al web de la institución. Esta opción puede que sea más adecuada para una intranet, donde el diseño y la navegación sencillos no son aspectos tan importantes como la exhaustividad. Se utiliza en Lisa (figuras 6 y 7), el catálogo de la biblioteca de la South Bank University $(S B U)$-que no debe confundirse con la base de datos del mismo nombre-

http://www.lisa.sbu.ac.uk/

Existen utilidades que ofrecen versiones más sofisticadas de creación de contenidos basadas en formularios web, como por ejemplo Flypage. Se trata de una serie de scripts en perl que permite al webmaster crear plantillas para que los usuarios introduzcan datos a través de un formulario web. El administrador tiene control completo sobre qué elementos pueden los usuarios cambiar en cada página. Flypage viene con un modelo de autorizaciones bastante completo, por lo que se pueden ofrecer distintos derechos a distintos usuarios.

http://www.flypage.com/features.html

\section{Gc con programación}

Si la biblioteca dispone de recursos para contratar un profesional informático que programe pequeñas aplicaciones se le abre un segundo nivel de gc con posibilidades muy interesantes. Por ejemplo, almacenar los datos en una bdd permite reutilizarlos en muchas páginas diferentes y actualizarlos sólo una vez ${ }^{1,2}$. Tener la información en una bdd tiene también otras ventajas: el sitio se pue- 

de rediseñar fácilmente; los no expertos pueden introducir información a través de formularios; y la estructuración de la base de datos obliga a una sana mayor disciplina para mantener la coherencia de los datos. Los sitios web basados en una bdd se parecen un poco a la misma, lo que en general causa buena impresión a los usuarios, aunque a algunos de ellos les puede parecer que los webs son menos "humanos".

Los datos de la bdd pueden ofrecerse de dos formas:

a) Salida como html estático.

Por ejemplo el sistema gestor de bdd Microsoft Access permite presentar html estático a partir de búsquedas o de tablas. El inconveniente es que el control sobre qué elementos de un registro se presentan es muy pobre y el formato dentro del mismo no se puede cambiar. La solución a esto es programar en Visual basic (VB) de manera que cuando se clique una opción específica, los datos pasen de la bdd a un fichero. De este modo se puede acceder a cualquier elemento de la bdd como una variable del programa. Idealmente se puede escribir datos desde la bdd a plantillas predefinidas en vez de codificar toda la página en VB, ya que ello supondría una tarea técnica complicada (o al menos laboriosa) para diseñar la presentación de los resultados. También se puede prever un botón para enviar la página al servidor por ftp. Una vez creado, este programa es bastante transparente y fácil de editar con unos conocimientos mínimos. Evidentemente, las páginas resultantes puede modificarlas cualquier persona familiarizada con html.

Ésta es la opción que se escogió para LTWorld, un servicio de información online para bibliotecarios realizado por Litc (Library and Information Technology Centre), de la South Bank University

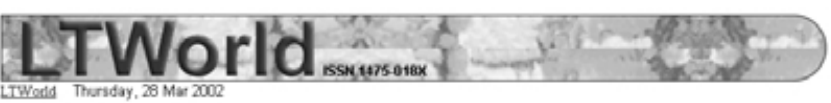

\section{Europe and culture, a new EU culture portal}

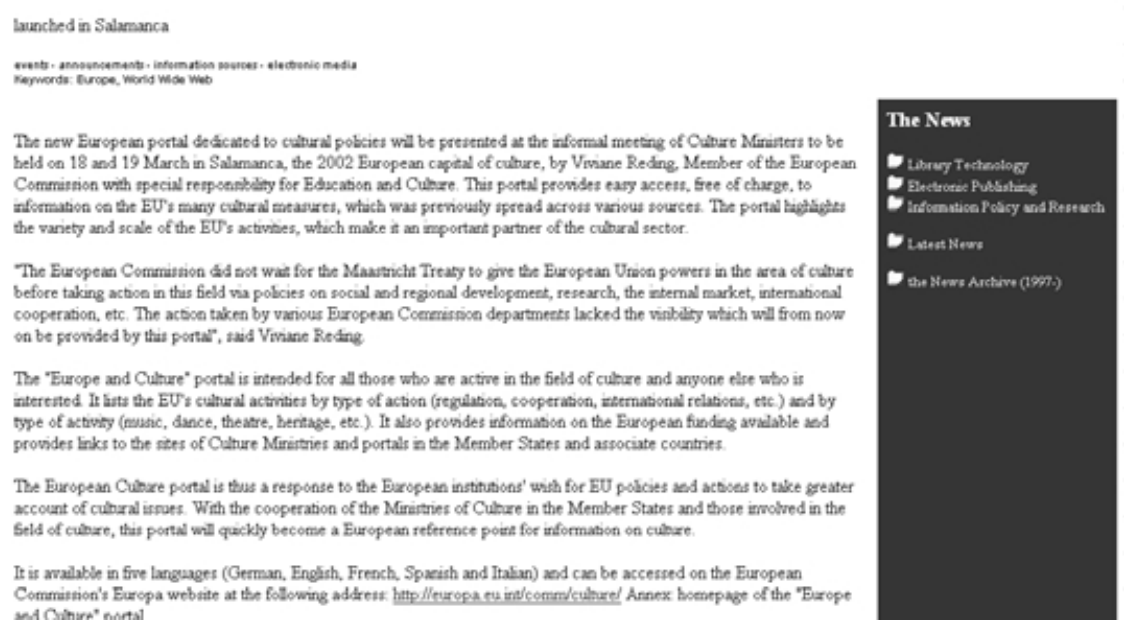

Fig. 5. Ejemplo de noticia de LTWorld

Please use this form to add an entry to the LISA News page.

Please remember that whatever you type here will go live on LISA as soon as you press the submit button. Please make sure all information is correct before submitting.

if you have any problems with this form, or submit incorrect information please notify the Web site co-ordinator ASAP.

Thanks

Title of Entry

Full URL (including http:I/) if

Required

Text of Entry

Your name and job title Submit Reset

Last Updated 21 March, 2001

Pages produced by LIS Web Team 92000

Fig. 6. Formulario en Access para introducir noticias en Lisa news.

Cualquier miembro del staff que lo desee puede añadir noticias fácilmente, rellenando y enviando el formulario (izquierda). Un script cgi procesa los datos de modo que el texto formateado aparece en la página de noticias (derecha) en html

- conocido centro especializado en temas bibliotecarios de brillante trayectoria desde sus inicios allá en los $80 \mathrm{~s}$ - Cualquier miembro de Litc puede agregar noticias llenando el formulario en Access (fig. 2) y publicarlas automáticamente clicando el botón que envía los datos al servidor por ftp.

http://www.lisa.sbu.ac.uk

http://www.sbu.ac.uk/litc/

Cuando se añade una noticia (fig. 5) a la bdd, automáticamente se generan los nuevos titulares y/o sumarios de las siguientes páginas:
1. Portada del propio Litc, que incluye las últimas novedades del mundo bibliotecario (fig. 1).

2. Portada de LTWorld (fig. 3).

3. Página de la sección de noticias —éstas están agrupadas por secciones- (fig. 4).

La bdd también crea un fichero RSS, un formato para sindicar contenidos (RDF site summary, siendo RDF las siglas de resource description format). Cuando es el caso, también genera un mensaje de correo-e para los usuarios suscritos a la sección o tema con el título y cabecera de la noticia. Son aplica- 
ciones simples, pero eficaces, que facilitan mucho el trabajo diario.

\section{b) Construir las páginas web "al momento"}

La segunda opción es conectar la bdd al sitio web de una forma dinámica, de forma que cuando un usuario pide una página en particular, ésta se crea al momento con los datos resultantes de la búsqueda en la bdd ${ }^{1,2}$

Gestionar información con una bdd es mucho más fácil y ofrece varias ventajas: los datos sólo tienen que actualizarse en la misma pero en cambio pueden visualizarse en distintos puntos y con múltiples formatos: generación de listas por materias, títulos, editoriales, etc. El inconveniente es que se tiene que saber programar SQL y tener acceso al servidor web para alojar la bdd en la misma máquina. Sin embargo el resultado es mucho más impactante. Véanse los webs de las bibliotecas de las universidades de Nottingham y Durham.

http://www.nottingham.ac.uk/is/

http://www.dur.ac.uk/library/

\section{Sistemas gestores de conte- nidos}

Actualmente en el mercado existen más de 300 sistemas gestores de contenidos (sgc) completamente desarrollados, aunque no imaginamos que muchas bibliotecas sientan aún la necesidad de usar alguno ${ }^{3,4}$. Sería deseable que los bibliotecarios participaran más activamente en la adquisición de un sgc no sólo para su biblioteca sino también, y sobre todo, para toda la organización ${ }^{5}$, ya que la mayor parte de sus conocimientos básicos son adecuados al caso: aplicación de metadatos (indizar, clasificar), cómo ofrecer acceso por materias, saber que los sistemas tienen que cumplir normativas abiertas para la interoperabilidad, conciencia de la importancia de tener en cuenta las necesidades del

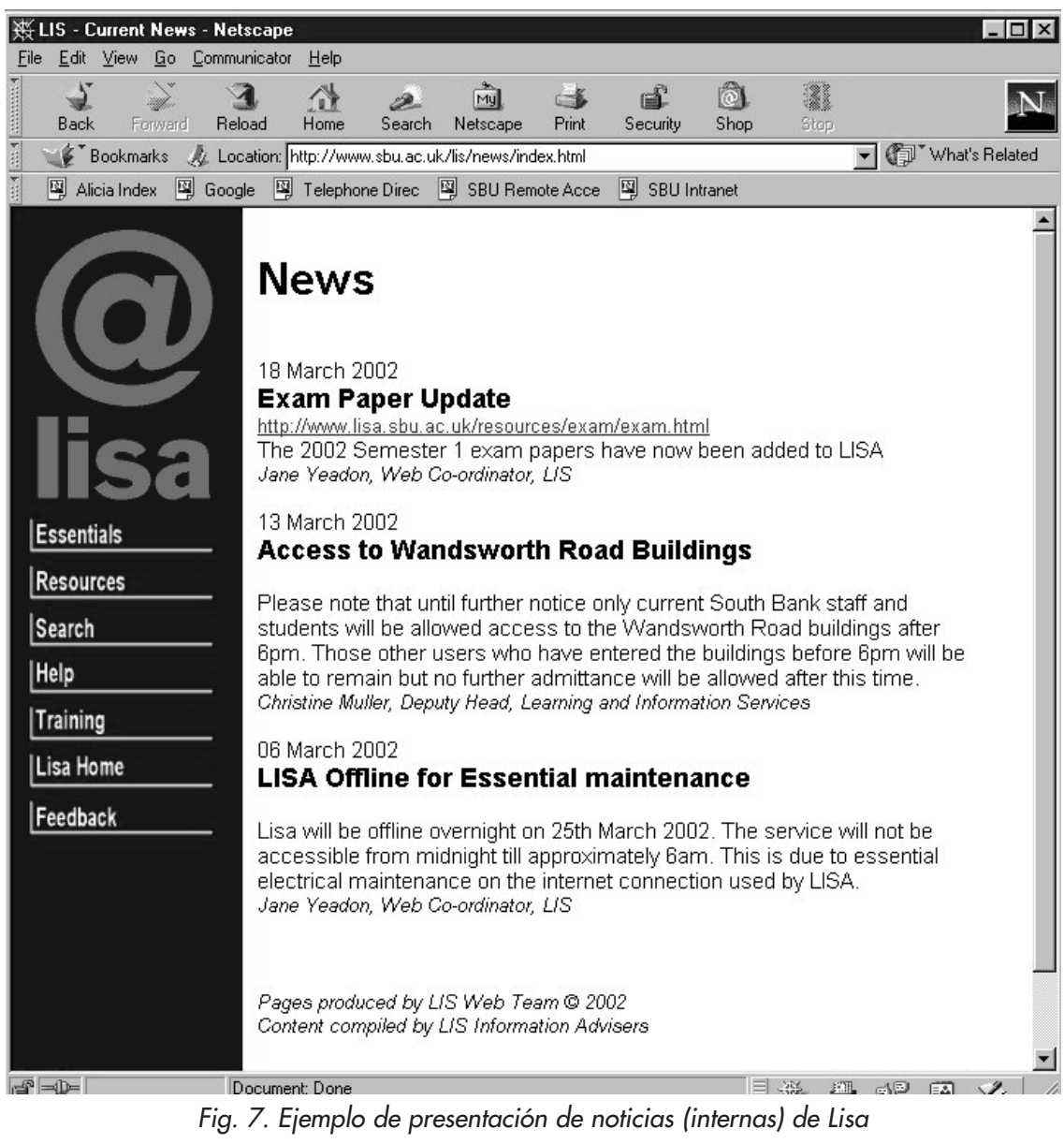

usuario final, intuir en muchos casos la necesidad de la intervención humana adicional, etc.

Poco a poco las bibliotecas irán teniendo sgcs. Por ejemplo el sistema XDirectory, de Esprit/Soutron, se presenta como sgc para bibliotecas y con él se pueden crear portales de información para la comunidad. Este producto permite al administrador delegar la alimentación de datos a las distintas personas situadas a lo largo de la cadena de la información que poseen o adquieren la más reciente y las noticias. La introducción de datos la realizan también a través de un formulario web.

Para quienes gestionan contenidos a gran escala, una tecnología prometedora es Zope $e^{6}$, que sigue la iniciativa Open Source (código fuente abierto), la cual se está desarrollando rápidamente y de forma cooperativa; el software se puede bajar de internet gratuitamente. La desventaja es que desarrollar aplicaciones no resulta trivial y requiere bastante formación y dedicación. Es discutible si el sistema cubrirá las expectativas de crear páginas asp a partir de una base de datos. Los sgc existen como un tipo de plataforma de lanzamiento de portales. Aquí hay un espacio para la cooperación interprofesional. Los bibliotecarios tendrían que unirse y trabajar juntos para incrementar el mutuo conocimiento compartido, y facilitar así, además, la entrada a nuevos usuarios que ejerzan en este área.

http://www.zope.com

http://www.opensource.org/

http://www.espritsoutronpartnersh ip.com/index.asp

\section{Bibliografía}

1. Gardner, Mike; Pinfield, Stephen. "Database-backed library websites: a case study of the use of PHP and MySQL at the University of Nottingham". En: Program, 2001, v. 35, n. 1, pp. 33-42.

2. Brabban, Philip; Kobasa, Paul. "Forget 'static' - get 'dynamic'! Using active server pa- 
ges to manage electronic resources". En: Vine, 2001, septiembre, n. 124 , pp. 27-35.

3. Browning, Paul; Lowndes, Mike. "JISC TechWatch Report: content management systems". En: JISC, 2001, noviembre.

http://www.jisc.ac.uk/techwatch/reports/tsw_O1 -02.pdf

4. Hunter, Philip. "A content management and web publishing systems gazetteer". En: Cultivate, 2001, noviembre.

http://www.cultivate-int.org/issue $5 / \mathrm{cms}$
5. White, Martin. "Content management systems for intranets". En: Vine, 2001, septiembre, n. 124 , pp. $46-50$

6. Ramsden, Anne; Turpie, David; Rea, Jonathan. "Managing the intranet: trying a new tool". En: Vine, 2001, septiembre, n. 124, pp. 36-45.

Texto original: Cox, Andrew; Yeadon, Jane. "Practical content management on the web: an overview". En: Online information 2001 procee- dings, 2001, diciembre, pp. 31-35.

Nota: Dirigir la correspondencia a:

Perry Library, South Bank University, 103 Borough Road, London SE1 $0 A A$

La traducción y adaptación de la comunicación anterior ha sido realizada para EPI por Clara Baiget baigetc@sbu.ac.uk baigetc@hotmail.com 